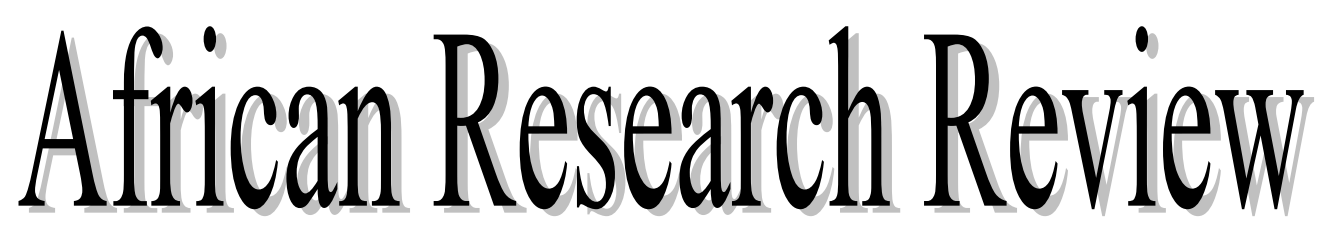

AN INTERNATIONAL MULTI-DISCIPLINARY JOURNAL,

BAHIR DAR, ETHIOPIA

AFRREV VOL. 11 (4), SERIAL NO. 48, SEPTEMBER, 2017:74-89

ISSN 1994-9057 (Print)

ISSN 2070-0083 (Online)

DOI : http://dx.doi.org/10.4314/afrrev.v11i4.7

\title{
Multivariate Analysis of Household Decision Making, Contraceptive Use and Fertility Behaviour among Ever- Married Men in Nigeria
}

\author{
Asa, S. S.; Kupoluyi, Joseph Ayodeji \\ Department of Demography and Social Statistics \\ Obafemi Awolowo University, Ile-Ife, \\ Osun State, Nigeria \\ E-mail: ayodejikupoluyi@gmail.com \\ Phone: $+234(0) 8037269871$
}

Oyinloye, B. O., Titilayo, A.

Department of Demography and Social Statistics

Obafemi Awolowo University, Ile-Ife,

Osun State, Nigeria

\begin{abstract}
The study examined the effect of household decision making on the use of contraceptives and fertility behaviour of ever-married men in Nigeria. Men's Recode Dataset of 2013 Nigerian Demographic and Health Survey (NDHS) dataset was used. A sample size of 8,981 ever married men aged 15-49 were analyzed using Chi-square test and multinomial logistic regression. The study revealed that majority of men who take a sole decision on their earning and are using contraceptives want more children. Other factors influencing men's fertility behaviour included age, education, wealth status, age at marriage, religion, the number of living children, ethnicity and media exposure. The study concluded that women empowerment in decision making and in the use of contraceptive methods should be promoted to reduce men's fertility behaviour.
\end{abstract}

Key Words: Contraceptive, decision making, fertility behaviour, ever-married Men, Nigeria

Copyright $\odot$ International Association of African Researchers and Reviewers, 2006-2017:

www.afrrevjo.net.

Indexed African Journals Online: www.ajol.info 
AFRREV VOL. 11 (4), S/NO 48, SEPTEMBER, 2017

\section{Introduction}

Lowering population growth rates has been the goal of many countries particularly, in sub-Saharan Africa (Alkema et al, 2013). Although there has been some decline in fertility in the region (Tabutin and Schoumaker, 2004), many countries like Nigeria still has a high fertility situation. The 2013 Nigerian Demographic and Health Survey (NDHS) reported an average of 5.2 children per woman at the end of her reproductive years. This reveals a little reduction from 5.5 children per woman reported for the years 2003 and 2008 (National Population Commission [(NPopC), Nigeria, and ICF International, $(2004 ; 2009 ; 2014)]$. This scenario has significantly contributed to the present population growth rate of $2.63 \%$ (World Bank, 2015) and including other negative implications on the economy of the country (Shapiro and Gebreselassie, 2007; Bongaarts, 2008; Moultrie et al., 2008; UNFPA, 2012). However, the slow decline in fertility experienced in the country has been attributed to low contraceptive use. Contraceptive use which gives individuals the ability to achieve their ideal family size through the prevention of unwanted and unplanned births is one of the most effective measures to reducing high fertility levels, as evident in the developed worlds (Frost, 2004; Graff, 2014). Although Nigeria has adopted various policies and programs aimed at reducing population growth rates, its impact on fertility has not been quite notable (Okezie et al, 2010). Currently, the modern contraceptive prevalence rate in Nigeria stands at $11 \%$, a $2 \%$ increase from what was obtained in 2008 (NPoC and ICF International, 2008; 2013).

Several studies on fertility issues and achieving lower population growth rates have focused mostly on women for the past many years including in Nigeria (Nicoletta et al, 2013; Lamidi, 2015; Charles et al, 2015; Van Deb Broeck and Maertens, 2015; Kana et al, 2016). Also, efforts in improving reproductive health outcomes through family planning and contraceptive use have centered mostly on women (Dewi, 2009) particularly in the African context. Yet, these efforts have not translated into a meaningful decline in fertility levels. This is because until recently, male involvement in reproductive health matters remains very limited and as such, has not been seen relevant in fertility- related issues (Caldwell and Caldwell, 1987; Oyediran et al, 2002; Akindele and Adebimpe, 2013). Similarly, majority of studies carried out on factors associated with contraceptive use and childbearing have overlooked men's crucial role and decision (Kaida et al, 2005; Ditekemena et al, 2012; Streefland et al, 2013), thereby making the idea of fertility performance a woman's sole concern (Mahmood and Ringheim, 1996; Wortham, 2002; Magadi and Curtis, 2003). Additionally, because it is generally seen that it is the woman that bear children, many studies conducted in past and even recently were presented as if men and women have similar fertility desires but which is not true in most cases.

Generally, reproduction does not involve only a woman. Giving birth involves two parties - a man and a woman. Thus, the need to examine the role of men in fertility issues is quite imperative. This statement was also emphasized at the 1994 International Conference on Population and Development (ICPD) in Cairo where it was declared that men should be involved in sexual and reproductive matters such as family planning and contraceptive use. Men, particularly in patriarchal settings like Nigeria, play a dominant role in influencing fertility choices in their homes. Like in many agrarian societies, Nigeria is characterized by large family size where men give birth to a large

Copyright () International Association of African Researchers and Reviewers, 2006-2017: www.afrevjo.net.

Indexed African Journals Online: www.ajol.info 
AFRREV VOL. 11 (4), S/NO 48, SEPTEMBER, 2017

number of children for meeting economic needs, family labour, and due to fear of death of a child (Caldwell, 1990; Blacker et al 2005). Such perceptions, however, are embedded in their cultural and societal values. But due to the men's dominant figure as a rule maker, ideas about having large family sizes daunt men's and couple's utilization of contraceptives. Thus, the success of family planning will be contingent upon men's approval (Bawah, 2002; Babalola et al, 2008; Ijadunola et al., 2010). To buttress this fact, a study carried out among married women by Oni and McCarty (1991) found that husbands' disapproval was the cause of non-use of contraceptives among those women.

Studies on fertility behaviour and contraceptive use have been found to be associated with men's socio-economic status- education and age at marriage (Martinez et al, 2011), marital status (Fuse, 2010), wealth status (Koj, 2000; Hinde and Mturi, 2000), region (Giusti and Vignoli,2006), type of family head, place of residence and religion (Uddin et al, 2011); gender roles (Kaufman 2000; Puur, Oláh et al 2008; Goldscheider et al, 2010), multi-partners (Carlson and Furstenberg 2006; Guzzo and Furstenberg, 2007), income (Guzzo and Furstenberg, 2007) and current number of living children (Lappegård and Rønsen, 2009; Kabagenyi et al, 2014; Kogay and Itua, 2017), among others. Yet, limited studies have delved into men's decision-making role in affecting contraceptive use, as a means of controlling fertility in Nigeria. Studies in sub-Saharan Africa have shown that male partners have absolute decision-making power that brings about the compliance and submission of their wives (Vouking, 2014). For instance, a study carried out among Mozambique women showed that husband influenced a woman's intention to use contraceptives (Mboane and Bhatta, 2015). Similar findings were also observed in Ghana, Zambia, Kenya and Uganda (Bawah et al, 1999; Blacker et al, 2015; Belohlav and Karra, 2013). Thus, this paper seeks to know the proportion of ever-married men taking final decision on contraceptive use, and by what type of contraceptive methods. It further seeks to know if there is a significant relationship between household decision making and contraceptive, household decision making and fertility behaviour and also, the interaction effect of household decision making and contraceptive use on fertility behaviour. Understanding these could be central in shaping interventions to address men's fertility behaviour.

\section{Data and Methods}

\section{Data}

The study used the Men's Recode Dataset of the 2013 Nigerian Demographic and Health Survey (NDHS) dataset. The 2013 NDHS is a three-stage sampling crosssectional dataset. The 2006 enumeration area (EA) census frame was employed in dividing the whole country into clusters (also known as Primary Sampling Units (PSU)). This led to the identification of a total of 904 PSU made up of 372 and 532 in urban and rural areas respectively. The use of Population Proportion to Size (PPS) to select 893 localities independently was involved in the first stage. The second stage involved a complete household listing for each PSU which in turn served as the sampling frame for the selected households interviewed. A random selection of 45 households from each urban and rural cluster using systematic sampling approach characterized the third stage. Half of the households selected was further sampled and all men aged 15-49 years in the household (who were either permanent residents or visitors who slept in the household the previous night before the survey) were eligible

Copyright (C) International Association of African Researchers and Reviewers, 2006-2017: www. afrrevjo.net.

Indexed African Journals Online: www.ajol.info 
to be interviewed (NPopC and ICF International, 2014). From all the 904 PSU the total weighted number of eligible respondents interviewed was 17, 359 out of which 8,981 ever married men were selected for this study.

\section{Measurement of Key Variables}

The outcome variable is fertility behaviour and was defined and measured in this study as wanting more children, want no more children and undecided. The principal explanatory variables include household decision making which was measured using decision making on respondent's earnings, healthcare, and large household purchase and were categorized as a husband only, wife only, jointly, and someone else/others, and contraceptive use which was recoded as "using" and "not using". Explanatory variables used in this study include age (15-19, 20-24, 25-29, 30-34, 35-39, 40-44, 4549), household wealth index (poorest, poorer, middle, richer, richest), place of residence (urban, rural), region of residence (North Central, North East, North West, South East, South-South, South-West), educational attainment (no education, primary, secondary, higher), number of living children $(0,1-4,5+)$, and children ever born $(0$, $1-2,3-4,5+)$. Other explanatory variables used but recoded for the suitability of this study include; type of contraceptive use (never use, folkloric, traditional, and modern method), religion (Christian, Islam, traditional/others), occupational status (working, not working), number of sexual partners $(0,1,2+)$, exposure to mass media (exposed and not exposed), Age at first marriage $(<20,20-24,25-49)$, number of wives (single, monogamous, polygamous), children ever born (0, 1-2, 3-4, 5+), and residing status of partner (living with partner and not living with partner).

\section{Conceptual Framework}

Figure 1 explained factors directly or indirectly influence fertility behaviour among ever-married men. Of note and interest are the interaction effect of household decisionmaking and contraceptive use and its effect on fertility behaviour among ever-married men.

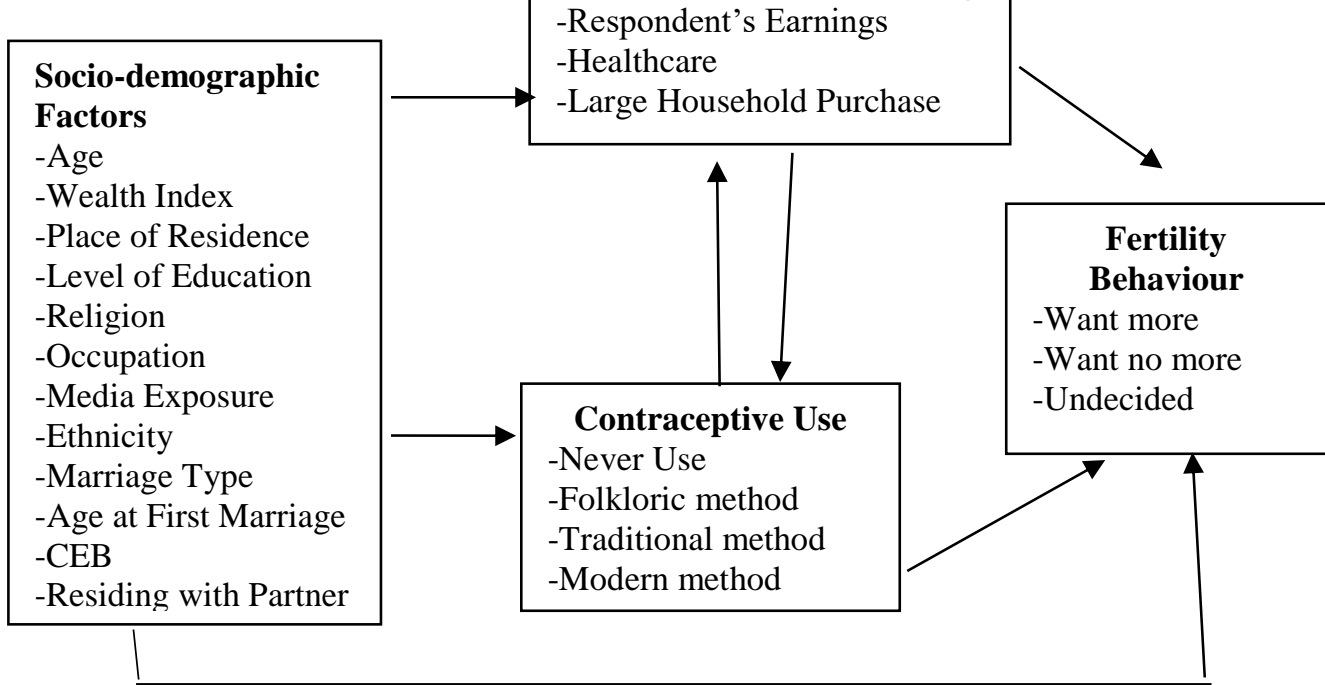

Figure 1: Conceptual framework showing direct and indirect link to the outcome variable (Fertility Behaviour)

Copyright (C) International Association of African Researchers and Reviewers, 2006-2017: www.afrrevjo.net.

Indexed African Journals Online: www.ajol.info 
AFRREV VOL. 11 (4), S/NO 48, SEPTEMBER, 2017

\section{Statistical Analysis}

Descriptive and inferential statistics were employed in the data analysis using STATA 12 statistical package (Stata Corp., 2012). To ensure reliable data, men's sample weights and STATA survey command (SVY) were applied to adjust for stratified sample design and the effect of over-sampling or under-sampling of some regions or areas. Characteristics of the respondents were described using percentage distribution while Pearson 's Chi-square tests were employed to establish level of significance and degree of association between explanatory variables and response variable. The Multinomial logistic regression model was fitted to assess factors associated with the relative risk ratios (RRR) of wanting more children, want no more children or undecided. This was done because the response variable is unordered categories with more than two outcomes. Check for multi-collinearity was performed to evaluate simple correlations among the independent variables before the fitting of the model. Variables that exceeds the threshold of the variance inflation factor (VIF) were dropped from the model while those that correlated with response variable were retained.

\section{Results}

Results in Table 1 showed that seven out of ten ever married men take sole decisions on how their earnings are spent while about one-fifth of the respondents take a joint decision with their partners. Less than $10 \%$ of the sampled men reported that their spouse takes a sole decision on how their earnings are spent. Sixty-five percent of the respondents reported that they take a sole decision on their healthcare while about onefifth took decisions about their healthcare jointly with their spouse. With regards to household purchases, $22.1 \%$ of the respondents reported taking joint decisions while about $23 \%$ reported that their spouse takes sole decisions. In all, the different areas of household decision making, the proportion reported to be by someone else or others is less than $1 \%$. Also, $83 \%$ of ever-married men were not using contraceptives while $17 \%$ were using contraceptives. The distribution of contraceptive use by type revealed however that respondents' spouses use contraceptives and the dominant method among male-based contraceptive was condom which constitutes $36.6 \%$ of respondents using contraceptives. In all, $10.2 \%$ have ever used modern contraceptive while respondents using Folkloric and Traditional method constitute about $1 \%$ and $6 \%$ respectively. The dominant method used by the respondents is condom and it constitutes about $37 \%$ of those who have ever used contraceptives. This is followed by periodic abstinence and withdrawal methods which constitute $21 \%$ and $16.4 \%$ respectively 
Table 1: Percentage Distribution of Decision making and Contraceptive Use among ever-married men age 15-49, Nigeria, 2013 DHS

\begin{tabular}{|c|c|c|}
\hline Household Decisions & Frequency & Percent \\
\hline $\begin{array}{l}\text { Respondent's earnings }(\mathrm{N}=7,965) \\
\text { Husband only } \\
\text { Wife only } \\
\text { Jointly } \\
\text { Someone else/others }\end{array}$ & $\begin{array}{l}5,825 \\
749 \\
1,370 \\
20\end{array}$ & $\begin{array}{l}73.1 \\
9.4 \\
17.2 \\
0.3\end{array}$ \\
\hline $\begin{array}{l}\text { Healthcare }(\mathrm{N}=8,690) \\
\text { Husband only } \\
\text { Wife only } \\
\text { Jointly } \\
\text { Someone else/others }\end{array}$ & $\begin{array}{l}5,668 \\
1,034 \\
1,920 \\
67\end{array}$ & $\begin{array}{l}65.2 \\
11.9 \\
22.1 \\
0.8\end{array}$ \\
\hline $\begin{array}{l}\text { Household purchases }(\mathrm{N}=8,981) \\
\text { Husband only } \\
\text { Wife only } \\
\text { Jointly } \\
\text { Someone else/others }\end{array}$ & $\begin{array}{l}4,361 \\
2,030 \\
2,265 \\
36\end{array}$ & $\begin{array}{l}50.2 \\
23.4 \\
26.1 \\
0.4\end{array}$ \\
\hline Contraceptive Use & & \\
\hline $\begin{array}{l}\text { Ever use Contraceptive }(\mathrm{N}=8981) \\
\text { Yes } \\
\text { No }\end{array}$ & $\begin{array}{l}1,531 \\
7,450\end{array}$ & $\begin{array}{l}17.1 \\
82.9\end{array}$ \\
\hline $\begin{array}{l}\text { Types of Contraceptive Ever Used } \\
\text { (N=1531) } \\
\text { No method } \\
\text { Folkloric method } \\
\text { Traditional method } \\
\text { Modern method }\end{array}$ & $\begin{array}{l}7,450 \\
44 \\
573 \\
914\end{array}$ & $\begin{array}{l}82.9 \\
0.5 \\
6.4 \\
10.2\end{array}$ \\
\hline $\begin{array}{l}\text { Contraceptives (N=1531) } \\
\text { Pill } \\
\text { IUD } \\
\text { Injections } \\
\text { Diaphragm } \\
\text { Condom } \\
\text { Female Sterilization } \\
\text { Male Sterilization } \\
\text { Periodic Abstinence } \\
\text { Withdrawal } \\
\text { Other } \\
\text { Implants/Norplant } \\
\text { Lactation amenorrhea (LAM) } \\
\text { Female condom } \\
\text { Foam or jelly } \\
\text { Other modern method }\end{array}$ & $\begin{array}{l}115 \\
33 \\
138 \\
5 \\
561 \\
4 \\
0 \\
321 \\
251 \\
44 \\
21 \\
19 \\
2 \\
3 \\
13\end{array}$ & $\begin{array}{l}7.5 \\
2.2 \\
9.0 \\
0.3 \\
36.6 \\
0.3 \\
0.0 \\
20.9 \\
16.4 \\
2.9 \\
1.4 \\
1.2 \\
0.1 \\
0.2 \\
0.9\end{array}$ \\
\hline
\end{tabular}

Copyright () International Association of African Researchers and Reviewers, 2006-2017: www.afrevjo.net. 
AFRREV VOL. 11 (4), S/NO 48, SEPTEMBER, 2017

\section{Bivariate}

Table 2 shows three areas of household decision making (respondents' earnings, health care, and large purchases) examined alongside contraceptive use. The result shows a significant association between the decision on respondents' earnings and contraceptive use $(\mathrm{p}<0.05)$. At least two in five respondents who take a sole decision on their earnings use contraceptives while among those who reported that their wives take a sole decision on their earnings, about $83 \%$ are using contraceptives. Similar patterns were observed with regards to healthcare and large purchases showing a significant association existed between household decision-making and contraceptive use $(\mathrm{p}<0.05)$

Table 2: Distribution of Respondents by Household Decision making and Contraceptive use

\begin{tabular}{|c|c|c|c|}
\hline \multirow[t]{2}{*}{ Decision making } & \multicolumn{2}{|c|}{ Contraceptive Use } & \multirow[t]{2}{*}{ Total } \\
\hline & Using & Not Using & \\
\hline \multicolumn{4}{|l|}{ Respondent's earnings } \\
\hline husband only & 3739 (85.7) & $622(14.3)$ & 4361 \\
\hline wife only & $1684(83.0)$ & $346(17.0)$ & 2030 \\
\hline jointly & $1775(78.4)$ & $490(21.6)$ & 2265 \\
\hline Someone else/others & $30(83.2)$ & $6(16.8)$ & 36 \\
\hline Total & $7228(83.2)$ & $1463(16.8)$ & $8691(100.0)$ \\
\hline \multicolumn{4}{|l|}{$X^{2}=66.18, p=0.0001$} \\
\hline \multicolumn{4}{|l|}{ Healthcare } \\
\hline husband only & 4787 (84.4) & $882(15.6)$ & 5668 \\
\hline wife only & $897(86.8)$ & $137(13.2)$ & 1034 \\
\hline jointly & $1483(77.2)$ & $438(22.8)$ & 1921 \\
\hline Someone else/others & $61(90.2)$ & $7(9.8)$ & 67 \\
\hline Total & $7227(83.2)$ & $1463(16.8)$ & $8690(100.0)$ \\
\hline \multicolumn{4}{|l|}{$X^{2}=66.18, p=0.0001$} \\
\hline \multicolumn{4}{|l|}{ Household Purchases } \\
\hline husband only & $3739(85.8)$ & $622(14.2)$ & 4361 \\
\hline wife only & $1684(83.0)$ & $346(17.0)$ & 2030 \\
\hline jointly & $1775(78.4)$ & $490(21.6)$ & 2265 \\
\hline Someone else/others & $30(83.2)$ & $6(16.8)$ & 36 \\
\hline Total & $7228(83.2)$ & $1463(16.8)$ & $8691(100.0)$ \\
\hline$X^{2}=56.88$ & & & \\
\hline
\end{tabular}

Result in Table 3 shows a significant association exists between contraceptive use and fertility behaviour $(p<0.05)$. Nearly $61 \%$ of those using contraceptives want more children while about a quarter of them want no more children. Also among those who are using contraceptives, the result reveals that the type of contraceptive methods used has a significant association with fertility behaviour $(\mathrm{p}<0.05)$. A large proportion of respondents $(80 \%)$ who are using modern contraceptive methods want more children. This may be suggestive of the fact that contraceptives are used more for birth spacing. Of interest is the fact that respondents who use any type of contraceptives still want more children. This implies that the use of contraceptives is not because they don't

Copyright () International Association of African Researchers and Reviewers, 2006-2017: www.afrevjo.net.

Indexed African Journals Online: www.ajol.info 
want more children. It is not surprising that about $90 \%$ of respondents who are not using any method want more children. Different areas of household decision making were found to be significantly associated with fertility behaviour $(\mathrm{p}<0.05)$. Among the respondents who take a sole decision on their earnings, about $85 \%$ want more children while among those who reported that their wives solely take a decision on respondents' earnings constitute about $84 \%$. Also among respondents who take a joint decision with their wives on respondent's earnings, about $75 \%$ want more children while about $23 \%$ want no more children. The same pattern is observed in respect of respondent's health and household purchases. The proportion of respondents who take a sole decision on healthcare and large purchases and wants more children to constitute $85 \%$ and $86 \%$ respectively.

Table 3: Distribution of respondents by contraceptive use, and fertility behaviour

Contraceptive Use

Fertility Behaviour (preference)

Total

\begin{tabular}{|c|c|c|c|c|}
\hline \multirow[t]{2}{*}{ Contraceptive Use } & \multicolumn{3}{|c|}{ Fertılity Behaviour (preference) } & \multirow[t]{2}{*}{ Total } \\
\hline & Want more & $\begin{array}{l}\text { Want no } \\
\text { more }\end{array}$ & others & \\
\hline $\begin{array}{l}\text { Contraceptive Use Status } \\
\text { Using } \\
\text { Not Using }\end{array}$ & $\begin{array}{l}941(61.5) \\
6288(84.4)\end{array}$ & $\begin{array}{l}382(24.9) \\
664(8.9)\end{array}$ & $\begin{array}{l}2089(13.6) \\
498(6.7)\end{array}$ & $\begin{array}{l}1531 \\
7450\end{array}$ \\
\hline Total & $7228(80.5)$ & 1047 (11.7) & $706(7.8)$ & 8981 \\
\hline \multicolumn{5}{|l|}{$X^{2}=433.05 p=0.0001$} \\
\hline $\begin{array}{l}\text { Type of Contraceptive Use } \\
\text { No method } \\
\text { Folkloric method } \\
\text { Traditional method } \\
\text { Modern method }\end{array}$ & $\begin{array}{l}241(90.5) \\
31(95.3) \\
78(92.9) \\
6879(80.0)\end{array}$ & $\begin{array}{l}9(3.5) \\
0(0.0) \\
2(2.9) \\
1035(12.0)\end{array}$ & $\begin{array}{l}16(6.0) \\
2(4.7) \\
4(4.2) \\
685(8.0)\end{array}$ & $\begin{array}{l}266 \\
32 \\
84 \\
8598\end{array}$ \\
\hline Total & $7228(80.5)$ & $1047(11.7)$ & $706(7.8)$ & $8981(100.0)$ \\
\hline \multicolumn{5}{|l|}{$X^{2}=33.05 \quad p=0.0279$} \\
\hline \multicolumn{5}{|l|}{$\begin{array}{l}\text { Decision making: } \\
\text { Respondent's earnings }\end{array}$} \\
\hline husband only & $4923(84.5)$ & $601(10.3)$ & $301(5.2)$ & 5825 \\
\hline wife only & $626(83.6)$ & $87(11.6)$ & $36(4.8)$ & 749 \\
\hline jointly & $987(72.1)$ & $320(23.3)$ & $63(4.6)$ & 1370 \\
\hline Someone else/others & $20(97.6)$ & $1(2.4)$ & $0(0.0)$ & 20 \\
\hline Total & $6556(82.3)$ & 1009 (12.7) & $400(5.0)$ & $7965(100.0)$ \\
\hline \multicolumn{5}{|l|}{$X^{2}=170.67 p=0.0001$} \\
\hline \multicolumn{5}{|l|}{ Respondent's Health } \\
\hline husband only & $4842(85.4)$ & $556(9.8)$ & $270(4.8)$ & 5668 \\
\hline wife only & $858(82.9)$ & $119(11.5)$ & $58(5.6)$ & 1034 \\
\hline jointly & $1450(75.5)$ & $366(19.1)$ & $105(5.4)$ & 1921 \\
\hline Someone else/others & $61(90.9)$ & $5(7.0)$ & $1(2.1)$ & 67 \\
\hline Total & $7211(82.9)$ & $1045(12.0)$ & $434(5.0)$ & 8690 \\
\hline \multicolumn{5}{|l|}{$X^{2}=122.70 p=0.0001$} \\
\hline \multicolumn{5}{|l|}{ Respondent's purchases } \\
\hline husband only & $3751(86.0)$ & $423(9.7)$ & $187(4.3)$ & 4361 \\
\hline wife only & $1687(83.1)$ & $234(11.5)$ & $109(5.4)$ & 2030 \\
\hline jointly & $1742(76.9)$ & $386(17.0)$ & $138(6.2)$ & 2265 \\
\hline Someone else/others & $32(90.6)$ & $2(5.4)$ & $1(4.0)$ & 36 \\
\hline Total & $7212(83.0)$ & $1045(12.0)$ & $434(5.0)$ & 8691 \\
\hline
\end{tabular}

$X^{2}=92.14 p=0.0001$ 
AFRREV VOL. 11 (4), S/NO 48, SEPTEMBER, 2017

\section{Multivariate Analysis}

Table 4 shows the result of multinomial logistic regression of the effect of contraceptive use, decision making and selected socio-economic and demographic factors on men's fertility behaviour. Result reveals that men who are using contraceptives are $27 \%$ less likely to want more children compared with men who are not using contraceptives, instead of wanting no more children. In addition, men who are using contraceptives are $92 \%$ less likely to be undecided to want more children compared with men who are not using contraceptives, instead of wanting no more children. Compared with men who take a decision on earning only, the likelihood of wanting more children instead of wanting no more children is $75 \%$ lower among men whose wives take decision alone than among men who take decision solely. In addition, men whose wife take a decision on earning only have $26 \%$ relative risk of undecided to have more children compared with those whose husband take a sole decision on earning, rather than wanting no more children. Similarly, the likelihood of men wanting more children instead of wanting no more children is 2.71 times more likely for men aged 20-24 than for men aged 15-19 (RC). Moreover, men aged 20-24 have 16.9 times higher likelihood of undecided to have more children relative to men aged 15-19, rather than wanting no more children. As regards educational level and in comparison, with men without education (RC), men with primary education were $59 \%$ lower of wanting more children as a replacement for want no more children. In addition, the likelihood of undecided to want more children instead of wanting no more children is $37 \%$ and $48 \%$ lower for men with primary and secondary education respectively than for men without education.

Table 4: Odds ratio based on multinomial logistic regression analysis, of contraceptive use, decision-making and fertility behaviour

\begin{tabular}{|c|c|c|c|c|}
\hline \multirow[t]{2}{*}{ Characteristics } & \multicolumn{2}{|c|}{$\begin{array}{l}\text { Want more children/ Want no } \\
\text { more children }\end{array}$} & \multicolumn{2}{|c|}{ Undecided / Want no more children } \\
\hline & Coefficient & $\begin{array}{l}{[95 \% \text { Conf. }} \\
\text { Interval }]\end{array}$ & Coefficient & [95\% Conf. Interval] \\
\hline $\begin{array}{l}\text { Contraceptive use } \\
\text { Not using (RC) } \\
\text { Using }\end{array}$ & $-0.73^{*}$ & $-0.91-0.56$ & 0.08 & $-0.20-0.35$ \\
\hline $\begin{array}{l}\text { Decision on earnings } \\
\text { husband only }(\mathrm{RC}) \\
\text { wife only } \\
\text { jointly } \\
\text { Someone else }\end{array}$ & $\begin{array}{l}-0.25^{*} \\
-0.16 \\
-0.06\end{array}$ & $\begin{array}{l}-0.49-0.01 \\
-0.58-0.26 \\
-2.65-2.54\end{array}$ & $\begin{array}{l}-0.74 \\
-0.45 \\
-12.56\end{array}$ & $\begin{array}{l}-1.14--0.32 \\
-1.10-0.20 \\
-2553.06-2527.92\end{array}$ \\
\hline $\begin{array}{l}\text { Decision on health } \\
\text { husband only(RC) } \\
\text { wife only } \\
\text { jointly } \\
\text { Someone else }\end{array}$ & $\begin{array}{l}-0.02 \\
-0.11 \\
-0.19 \\
\end{array}$ & $\begin{array}{l}-0.23-0.27 \\
-0.45-0.26 \\
-1.68-1.30 \\
\end{array}$ & $\begin{array}{l}-0.06 \\
0.19 \\
-14.00\end{array}$ & $\begin{array}{l}-0.45-0.34 \\
-0.37-0.75 \\
-2133.67-2105.66\end{array}$ \\
\hline $\begin{array}{l}\text { Decision on purchases } \\
\text { husband only (RC) } \\
\text { wife only } \\
\text { jointly } \\
\text { Someone else }\end{array}$ & $\begin{array}{l}-0.08 \\
0.03 \\
-0.96\end{array}$ & $\begin{array}{l}-0.29-0.13 \\
-0.20-0.27 \\
-3.00-1.07\end{array}$ & $\begin{array}{l}0.24 \\
-0.03 \\
-13.80\end{array}$ & $\begin{array}{l}-0.09-0.56 \\
-0.41-0.34 \\
-2683.49-2655.89\end{array}$ \\
\hline $\begin{array}{l}\text { Age } \\
15-19(\text { RC) } \\
20-24\end{array}$ & $2.71 *$ & $0.26-5.15$ & 16.86 & $-3443.40-3477.13$ \\
\hline
\end{tabular}

Copyright (C) International Association of African Researchers and Reviewers, 2006-2017: www.afrrevjo.net.

Indexed African Journals Online: www.ajol.info 


\begin{tabular}{|c|c|c|c|c|}
\hline $\begin{array}{l}25-29 \\
30-34 \\
35-39 \\
40-44 \\
45-49\end{array}$ & $\begin{array}{l}1.75 \\
1.13 \\
0.58 \\
0.03 \\
-0.33\end{array}$ & $\begin{array}{l}-0.44-3.94 \\
-1.05-3.30 \\
-1.59-2.75 \\
-2.14-2.21 \\
-2.51-1.85 \\
\end{array}$ & $\begin{array}{l}15.83 \\
15.57 \\
15.44 \\
15.14 \\
14.87 \\
\end{array}$ & $\begin{array}{l}-3444.44-3476.10 \\
-3444.69-3475.83 \\
-3444.82-3475.71 \\
-3445.12-3475.41 \\
-3445.39-3475.14 \\
\end{array}$ \\
\hline $\begin{array}{l}\text { Educational level } \\
\text { None (RC) } \\
\text { Primary } \\
\text { Secondary } \\
\text { Tertiary }\end{array}$ & $\begin{array}{l}-0.41^{*} \\
-0.30 \\
-0.31\end{array}$ & $\begin{array}{l}-0.76--0.05 \\
-0.66-0.05 \\
-0.70-0.05\end{array}$ & $\begin{array}{l}-0.63 * \\
-0.52 * \\
-0.53\end{array}$ & $\begin{array}{l}-1.12--0.16 \\
-1.02--0.04 \\
-1.07-0.01\end{array}$ \\
\hline $\begin{array}{l}\text { Wealth quintile } \\
\text { Poor (RC) } \\
\text { Poorer } \\
\text { Middle } \\
\text { Richer } \\
\text { Richest }\end{array}$ & $\begin{array}{l}-0.89^{*} \\
-1.13^{*} \\
-1.47^{*} \\
-1.75^{*}\end{array}$ & $\begin{array}{l}-1.39--0.40 \\
-1.62--0.64 \\
-1.97--0.98 \\
-2.27--1.23\end{array}$ & $\begin{array}{l}-0.10 \\
-0.29 \\
-0.60 \\
-0.86^{*}\end{array}$ & $\begin{array}{l}-0.77-0.57 \\
-0.96-0.38 \\
-1.30-0.09 \\
-1.61--0.12\end{array}$ \\
\hline $\begin{array}{l}\text { Religion } \\
\text { Christian (RC) } \\
\text { Islam } \\
\text { Others }\end{array}$ & $\begin{array}{l}1.03^{*} \\
0.59\end{array}$ & $\begin{array}{l}0.81-1.25 \\
-0.05-1.24\end{array}$ & $\begin{array}{l}0.58^{*} \\
0.32\end{array}$ & $\begin{array}{l}.24-0.93 \\
-0.73-1.39\end{array}$ \\
\hline $\begin{array}{l}\text { Place of } \\
\text { residence } \\
\text { Urban }(\mathbf{R C}) \\
\text { Rural }\end{array}$ & 0.08 & $-0.11-0.26$ & -0.23 & $-0.53-0.07$ \\
\hline $\begin{array}{l}\text { Ethnicity } \\
\text { Hausa/Fulani (RC) } \\
\text { Igbo/Ibo } \\
\text { Yoruba } \\
\text { Others }\end{array}$ & $\begin{array}{l}-1.62 * \\
-1.99 * \\
-1.28 *\end{array}$ & $\begin{array}{l}-2.05--1.19 \\
-2.38--1.60 \\
-1.66--0.90\end{array}$ & $\begin{array}{l}-0.89 * \\
-1.72 * \\
-1.05^{*}\end{array}$ & $\begin{array}{l}-1.49--0.30 \\
-2.27--1.17 \\
-1.54--0.55\end{array}$ \\
\hline $\begin{array}{l}\text { Number of Living } \\
\text { Children } \\
\text { None (RC) } \\
\text { 1-4 children } \\
5 \text { children and above }\end{array}$ & $\begin{array}{l}-1.53 * \\
-2.66 *\end{array}$ & $\begin{array}{l}-2.17--0.90 \\
-3.31--2.00\end{array}$ & $\begin{array}{l}-1.28 * \\
-1.84 *\end{array}$ & $\begin{array}{l}-2.06--0.49 \\
-2.67--1.01\end{array}$ \\
\hline $\begin{array}{l}\text { Age at marriage } \\
15-19(\text { RC) } \\
20-24 \\
25-49\end{array}$ & $\begin{array}{l}0.41^{*} \\
0.80^{*}\end{array}$ & $\begin{array}{l}0.15-0.66 \\
0.55-1.05\end{array}$ & $\begin{array}{l}0.18 \\
0.42 *\end{array}$ & $\begin{array}{l}-0.21-0.57 \\
0.03-0.82\end{array}$ \\
\hline $\begin{array}{l}\text { Media exposure } \\
\text { Not exposed (RC) } \\
\text { Exposed }\end{array}$ & $-0.24 *$ & $-0.42--0.08$ & -0.17 & $-0.45-0.12$ \\
\hline _cons & $5.56^{*}$ & $3.36-7.76$ & -12.80 & $-3473.07-3447.46$ \\
\hline \multicolumn{5}{|c|}{$\begin{array}{l}\text { Model summary: Log Likelihood }=-3551.55, \text { model chi- } \text { square }(\chi 2)=2063.67, d f=68, n=7744, p- \\
\text { value }<0.0000\end{array}$} \\
\hline
\end{tabular}

Regarding wealth quintile and in comparison, with poor men (RC), the likelihood of wanting more children instead of wanting no more children is $1.13,1.47$, and 1.75 times more likely for men in the middle, richer, and richest of wealth status respectively and $11 \%$ lower for poorer men. In addition, the likelihood of undecided to want more children rather than want no more children is $14 \%$ lower for the richest men than poor men (RC). On religion, the result shows that the relative log odds of wanting more children in preference to want no more children are 1.03 times more likely for Muslims than for Christians (RC). In addition, the likelihood of undecided to want more children instead of wanting no more children is $42 \%$ lower for Muslim than for Christian (RC). In comparison with the Hausa/Fulani ethnic group (RC), Igbo/Ibo, Yoruba and others

Copyright (C) International Association of African Researchers and Reviewers, 2006-2017: www.afrrevjo.net.

Indexed African Journals Online: www.ajol.info 
ethnic group were $1.62,1.99,1.28$ times respectively more likely to want more children as an alternative to wanting no more children. In addition, the likelihood of undecided for more children instead of wants no more children is $11 \%$ lower for Igbo and 1.72, 1.05 times more likely for Yoruba, and others ethnic groups than the Hausa/Fulani.

The multinomial result also revealed that the likelihood of want more children in place of want no more children is 1.53 times higher for men with 1-4 children and 2.66 times higher for men with 5 and above children than for men without a child. In addition, and in comparison, with men with no children (RC), men with 1-4 children and 5 and above children were 1.28 and 1.84 times higher to undecided for more children instead of wanting no more children. Furthermore, the result shows that the expected likelihood of want more children as a replacement to want no more children is 59\% and $20 \%$ lower for men who married at aged 20-24 and 25-49 respectively than for men aged 15-19 (RC). In addition, and in comparison, with men who married at aged 15-18 (RC), the likelihood of undecided for more children in preference to wanting no more children is $58 \%$ less likely for men who married at aged 25-49. Finally, the likelihood of want more children in preference to want no more children is $76 \%$ lower for men who were exposed to the mass media than for men who are not exposed to mass media (RC).

\section{Discussion}

The study examined the effect of household decision-making and contraceptive use on fertility behaviour among ever-married men in Nigeria. The results show that majority of ever-married men were not using contraceptive methods. A number of studies (MacQuarrie et al., 2015; Islam, 2013) have reported similar findings. In the study area, childbearing is culturally accepted within marriage and it is expected that a large proportion of married men who desired for more children will not use contraceptives. Also, poor promotion of male-controlled methods may be responsible for the finding since the use of contraceptive methods is considered as women's affairs. Likewise, the study found that condom was the dominant method among men using contraceptives. MacQuarrie et al. (2015) reported similar outcome using DHS data from 58 men's surveys in 18 countries in Africa, Asia, and Latin America and the Caribbean. They found that condom contributes the largest share to overall contraceptive use reported by men. This may be due to the fact that men used a condom for spacing and prevention of sexually transmitted diseases. The study further showed that three out of every five men who are using contraceptives want more children while only about a quarter want no more children. Similar results were reported by Kabagenyi et al. (2013); Islam, (2013); Hossain et al. (2009). The findings may be due to the fact that most married men use contraceptives more for birth spacing and not because they do not want more children. Multinomial analysis showed that majority of men who take a sole decision on their earnings want more children than those who reported that their wives take a sole decision on their earning. A similar finding has been reported in developing countries particularly in Sub-Saharan African. Vouking (2014); Okigbo et al. (2013); Odu et al. (2005) reported in their studies that men who solely take a decision on household earnings were more likely to want more children than men whose wives solely take a decision on household earnings. This finding may be due to dominating of men in reproductive decision making. Thus, men's reproductive ambition to a large extent affects their wives' reproductive behaviour in terms of family size, the timing of pregnancies and the use or non-use of contraceptives Men in patriarchal African

Copyright (C) International Association of African Researchers and Reviewers, 2006-2017: www.afrevjo.net.

Indexed African Journals Online: www.ajol.info 
societies demand more children than their wives. Greater economic input in family upkeep may also influence men decision. In Nigeria, men wanted more children than their wives and are motivated by the socio-economic benefits, a continuation of the family name and as a way of boosting the man's ego.

Analysis of the background characteristics of respondents revealed that age, age at marriage, wealth status, education, religion, ethnicity, the number of living children and media exposure influenced fertility behaviour. Men aged 20-24 want more children than men aged 15-19. This is expected because men aged 20-24 are more likely to want more children than single men. This finding corroborates Okigbo (2015) that single younger men used contraceptives to avoid children than men in their prime age (20-24) who want children to prove their fertility and marriage. Likewise, men's education plays an important role in fertility behaviour. It is expected that higher level of education will promote the use of contraceptives particularly the modern contraceptives which will influence fertility behaviour. Thus, the study reported higher odd for more children among men with no education compared with men with any form of education. This finding is in line with previous studies by Shahjahan et al. (2013); Kabagenyi et al. (2013); MacQuarrie et al. (2015); and Islam (2013) which reported that educated men often have smaller family size. This finding has been established. On the average, educated men tend to have smaller families because of the financial consequences of large families, tend to marry later and adopt the western culture which favours a small family size. In addition, they were more exposed to mass media such as radio, television, and newspapers which influenced their fertility behaviour of not desiring for more children than non-educated men.

This study also revealed that wealth status of men influenced their fertility behaviour. The study found a positive association between wealth quintile and fertility behaviour. The likelihood of wanting more children is higher among men with higher levels of wealth compared to the poor. Similar studies have been reported by Belohlav et al. (2016); Kana et al. (2016); MacQuarrie et al, 2015); Kabagenyi et al. (2013); Islam (2013); Martinez et al. (2012); Koji (2000); Hinde, and Mturi (2000); Uddin et al. (2011). They found that the likelihood of using contraceptives and having small family size was lower among rich men compared to those men in lowest wealth quintile. Childbearing is acceptable within marriage. This study showed that men who married at younger ages want no more children than men who married at older ages. This finding is in line with Martinez et al. (2011). This finding may be due to the fact that men who married at younger ages might have completed the desired family size earlier than men who married at older ages. Furthermore, the study found that the Muslims have a higher likelihood of wanting more children than for Christian. This finding is supported by Uddin et al. (2011). Christianity promotes one man one wife and therefore tends to have smaller family size. This is because the competition to produce the greater number of children among wives is lower among the Christians than the Muslims. Also, men who have had at least one child were more likely to want no more children. This is expected and is consistent with other studies (Lappegård and Rønsen, 2009; Kabagenyi et al, 2014; Kogay and Itua, 2017). The study revealed that Yoruba ethnic group had a lower desire for more children than other ethnic groups. They also have higher odds of wanting no more children than other ethnic groups. This is consistent with previous studies by Wusu and Isiugo-Abanihe (2006). The level of education, media exposure may have influenced the relationship. Finally, available evidence from

Copyright (C) International Association of African Researchers and Reviewers, 2006-2017: www.afrrevjo.net.

Indexed African Journals Online: www.ajol.info 
previous studies has shown that exposure to mass media messages promotes family planning which in turn may affect individual's contraceptive behaviour and desire for fewer children. This study revealed that men who were exposed to the mass media had a lower likelihood to want more children than men without media exposure. This was also observed by Gupta et al. (2003), Ajaero et al. (2016).

\section{Strengths and Limitations}

Decision making in fertility and contraceptive use is a complex process. Decisionmaking autonomy in the 2013 NDHS men's record dataset was measured using three types of household decisions: their own health care, making major household purchases, and visits to family or relatives. However, this study would have preferred to have direct data on decision making on contraceptive use and the type of contraceptive methods used rather than the proxy indicators used in this study.

\section{Conclusion}

This study found that decision-making and contraceptive use influenced fertility behaviour among ever-married men in Nigeria. Age, education, wealth status, age at marriage, religion, ethnicity, the number of living children and exposure to mass media were among the predictors of fertility behaviour of ever-married men in Nigeria. The study suggested that women empowerment in decision making and in the use of contraceptive methods should be promoted to reduce men's fertility behaviour.

\section{References}

Ajaero, C. K., Odimegwu, C., Ajaero, I. D. \& Nwachukwu, C. A. (2016). Access to mass media messages, and use of family planning in Nigeria: a spatiodemographic analysis from the 2013 DHS. BMC Public Health, 16 (427): 110. DOI: $10.1186 / \mathrm{s} 12889-016-2979-\mathrm{z}$.

Babalola, S., Folda, L. \& Babayaro, H. (2008). The effects of a communication program on contraceptive ideation and use among young women in Northern Nigeria. Stud. Fam.Plann., 39(3):211-220.

Bawah, A. A., Akweongo, P, Simmons, R. \& Phillips, J. (1999). Women's fears and men's anxieties: The impact of family planning on gender relations in Northern Ghana. Stud Fam Plann, 30(1). 54-66

Bawah, A. A. (2002). Spousal communication and family planning behaviour in Navrongo: A longitudinal assessment. Stud. Fam. Plann., 33(2). 185-194.

Belohlav, K. and Karra, M. (2013). Household decision making and contraceptive use in Zambia. Washington, DC: PopPov Network Policy Brief, Population Reference Bureau p.1-6. http://www.prb.org/pdf13/zambia-householddecisionmaking-contraception.pdf. Assessed 27th February 2016.

Blacker, J., Opiyo, C., Jasseh, M., Sloggett, A. \& Ssekamatte-Ssebuliba, J. (2005). Fertility in Kenya and Uganda: A comparative study of trends and determinants. Popul Stud, 59(3). 355-373.

Caldwell, C. (1990). High Fertility in Sub-Saharan Africa. Sci Am, 262(5). 118.

Caldwell, J. C., and Caldwell, P. (1987). The cultural context of high fertility in SubSaharan Africa. Popul Dev Rev, 13(3). 409-437. 
Carlson, M. J., \& Furstenberg, F. F. (2006). The prevalence and correlates of multipartnered fertility among urban U.S. parents. Journal of marriage and the family, 68(2). 718-732.

Ditekemena, J., Koole, O., Engmann, C., Matendo, R., Tshefu, A., Ryder, R. \& Colebunders, R. (2012). Determinants of male involvement in maternal in child health services in Sub- Saharan Africa: a review. Reprod Health, 9(1):32.

Frost, J. J., \& Finer, L. B. (2004). Factors associated with contraceptive use and nonuse in the United States. Perspect Sex Reprod Health, 39:90-99.

Fuse, K. (2010). Variations in attitudinal gender preferences for children across 50 lessdeveloped countries. J Demogr Res. 2010, 23 (36): 1031-1048.

Giusti, C. \& Vignoli, D. (2006). Determinants of contraceptive Use in Egypt: A Multilevel Approach. Stat Meth Appl. 15 (1): 89-106.

Goldscheider, F., Oláh, L. S. \& Puur, A. (2010). Reconciling studies of men's gender attitudes and fertility: Response to Westoff and Higgins. Demographic Research 22:189-198

Gupta, N., Katende, C., \& Bessinger, R. (2003). Associations of mass media exposure with family planning attitudes and practices in Uganda. Studies in Family Planning, Vol. 34, Issue. 1, p.19-31.

Guzzo, K. B., and Furstenberg, F.F. (2007). Multipartnered fertility among American Men. Demography 44(3):583-601.

Hinde, A. and Mturi, A. (2000). Recent trends in Tanzanian fertility. Popul Stud, 54 (2): 177-191.

Hossain, M. B., Phillips, J. F. \& Mozumder A. B. (2009). The effect of husband's fertility preferences on couple's reproductive behavior in rural Bangladesh. $J$ Biosoc Sci; 39: 745-57.

Kabagenyi, A., Ndugga, P., Wandera, S. \& Kwagala, B. (2014). Modern contraceptive use among sexually active men in Uganda: Does discussion with a health worker matter? BMC Public Health, 14(286).

Kaida. A., Kipp, W., Hessel, P. \& Konde-Lule, J. (2005). Male participation in family planning: results of a qualitative study in Mpigi District, Uganda. J Biosoc Sci, $37: 269-286$.

Kana, M. K., Tagurum, Y. O., Hassan, Z. I., Afolaranmi, T. O., Ogbeyi, G. O., Difa, J. A., Amede, P. A. \& Chirdan, O. O. (2016). Prevalence and determinants of contraceptive use in rural North-Eastern Nigeria: Results of a mixed qualitative and quantitative assessment. Annals of Nigerian Medicine, 10(1): 3-10.

Kaufman, G. 2000. Do gender role attitudes matter? Family Formation and dissolution among traditional and egalitarian men and women. Journal of Family Issues, 21: $128-144$.

Koc, J. (2000). Determinants of contraceptive use and method choice in Turkey. $J$ Biosoc Sci.32: 329-342.

Copyright () International Association of African Researchers and Reviewers, 2006-2017: www.afrrevjo.net.

Indexed African Journals Online: www.ajol.info 
AFRREV VOL. 11 (4), S/NO 48, SEPTEMBER, 2017

Kogay, V. \& Itua, I. (2017). Prevalence and socio-economic factors determining use of modern contraception among married men in Kyrgyzstan: Evidence from a demographic and health survey. Public Health, 142:56-63.

Lamidi, E. O. (2015). State variations in women's socioeconomic status and use of modern contraceptives in Nigeria. PLoS ONE 10(8): e0135172. DOI:10.1371/journal.pone.0135172.

Lappegård, T., M. Rønsen, \& Skrede, K. (2011). Fatherhood and fertility. Fathering 9(1) 103-120.

Magadi, M. A. \& Curtis, S. L. (2003). Trends and determinants of contraceptive method choice in Kenya. Stud Fam Plann, 34(3):149-159.

Mahmood, N. \& Ringheim, K. (1996). Factors affecting contraceptive use in Pakistan. Pakistan Dev Rev, 35(1):1-22.

Martinez, G., Daniels, K. \& Chandra, A. (2012). Fertility of men and women aged 1544 years in the United States: National Survey of Family Growth, 2006-2010, Natl Health Stat Rep. 51:1-29.2. Accessed 26th February, 2017 from https://www.cdc.gov/nchs/data/nhsr/nhsr051.pdf.

Mboane, R. \& Bhatta, M. (2015). Influence of husband's decision-making role on a woman's intention to use contraceptives among Mozambican women. Reproductive Health, 12:36. DOI: 10.1186/s12978

Odu, O. O., IJadunola, K. T. \& Parakoyi, D. B. (2005). Reproductive behaviour and determinants of fertility among men in a semi-urban Nigerian community. Journal of Community Medicine and Primary Health Care. 17(1):13-19

Oyediran, K. A, Ishola, G. P. and Feyisetan, B. J. (2002). Factors affecting evermarried men's contraceptive knowledge and use in Nigeria. J Biosoc Sci. 34(4):497-510.

Puur, A., Oláh, L. S., Tazi-Preve, M. I. \& Dorbritz, J. (2008). Men's childbearing desires and views of the male role in Europe at the dawn of the 21 st century. Demographic Research, 19: 1883-1912.

Shahjahan. M., Mumu, S. J., Afroz, A., Chowdhury, H. A., Kabir, R. \& Ahmed, K. (2013). Determinants of male participation in reproductive healthcare services: a cross-sectional study. Reprod Health; 10(1):27.

Stata Corp. (2012). Stata 12 College Station, Tx: StataCorp LP.

Streefland, P., Vassall, A., Bagdadi, S., Bashour, H., Zaher, H., Maaren, P., Kamminga, E., Wegelin-Schuringa, M., Van Liere, M. \& Murphy, C. (2013). Programming for Male Involvement in Reproductive Health. Report of the Meeting of WHO Regional Advisors in Reproductive Health WHO/PAHO Washington DC USA 5-7 September 2001. Vaccine, 21(12):1304-1309.

Tabutin, D. and Schoumaker, B. (2004). The demography of Sub-Saharan Africa from the 1950s to the 2000s. A survey of changes and a statistical assessment. Population (English Edition), 59: 457-519.

Copyright () International Association of African Researchers and Reviewers, 2006-2017: www.afrevjo.net.

Indexed African Journals Online: www.ajol.info 
Uddin, I., Bhuyan, K. and Islam, S. (2011). Determinants of desired family size and children ever born in Bangladesh. The Journal of Family Welfare, 57(2):3947.

UNFPA (2012) State of the World population 2012; By choice not by chance, family planning, human rights and development. New York: United Nations Population Fund. https://www.unfpa.org/sites/default/files/pubpdf/EN_SWOP2012_Report.pdf Assessed 05/09/17

Vouking, M. Z., Evina, C. D. \& Tadenfok, C. N. (2014). Male involvement in family planning decision making in Sub-Saharan Africa- What the evidence suggests. The Pan African Medical Journal, 19: 349. DOI: 10.11604/pamj.

Wasao, S. (2011). A comparative analysis of the socio-economic correlated of fertility in Cameroon and the Central African Republic. Prospects for fertility decline in high fertility countries. New York, 5-11. Retrieved 26th February 2017 from http://Www.Un.Org/Esa/Population/Publications/Prospectsdecline/Wasao.Pd f.

World Bank (2015). Population growth (Annual \%) in Nigeria. http://www.tradingeconomics.com/nigeria/population-growth-annualpercent-wb-data.html. Assessed 27th February 2017.

Wortham, R. A. (2002). Spatial differences in fertility decline in Kenya: Evidence from recent fertility surveys. Soc SciJ, 39(2):265-276.

Wusu, O. \& Isiugo-Abanihe, U. C. (2006). Interconnections among changing family structure, child rearing, and fertility behaviour among the Ogu, South-western Nigeria: A qualitative study. Demographic Research: Volume 14, Article 8 Pp 139-156 http://www.demographic-research.org/Volumes/Vol14/8/ DOI: 10.4054/DemRes.2006.14.8

National Population Commission (NPC) [Nigeria] and ICF International (2014). Nigeria demographic and health survey 2013. Abuja, Nigeria, and Rockville, Maryland, USA: NPC and ICF International.

National Population Commission [Nigeria] \& ORC Macro (2004). Nigeria demographic and health survey 2003. Calverton, Maryland, USA: National Population Commission and ORC Macro.

National Population Commission [Nigeria] \& ICF Macro (2009). Nigeria demographic and health survey 2008. Abuja, Nigeria: National Population Commission and ICF Macro. 